\title{
Determination of Nonlinear Refractive Indices by External Self-Focusing
}

\author{
B. Meier and A. Penzkofer
}

Naturwissenschaftliche Fakultät II - Physik, Universität, D-8400 Regensburg, Fed. Rep. Germany

Received 15 June 1989/Accepted 27 July 1989

\begin{abstract}
The nonlinear refractive index of benzene is determined by measuring the reduction of the beam divergence of picosecond ruby laser pulses when passing through a benzene sample. Time-integrated spatial beam profiles give an effective refractive index while time-resolved beam profiles measured with a streak camera allow the determination of the temporal evolution of the nonlinear refractive index.
\end{abstract}

PACS: $42.65,42.65 . J$

At high laser powers the refractive index of materials becomes intensity dependent. The spatial laser beam profile causes a spatial refractive index profile and leads to self-focusing [1-6]. The overall beam profile is responsible for whole-beam self-focusing while an intensity modulation of the spatial intensity distribution leads to a beam break-up (small-scale selffocusing) [5-8]. The combined effects of self-focusing and beam diffraction result in filament formation [1-5, 9-12]. The self-focusing increases the pulse intensity enormously and enhances all nonlinear optical effects [13-19]. The abrupt rise of nonlinear optical effects is an indication of self-focusing and may be used to determine the self-focusing length. The determination of the nonlinear refractive index from the abrupt rise of nonlinear optical effects is complicated by the fact that either whole-scale self-focusing or small-scale selffocusing may act and the small-scale self-focusing parameters (ripple widths and modulation depths) are difficult to determine.

The vagueness of whole-scale or small-scale selffocusing dynamics may be avoided by changing from internal self-focusing (focal point caused by nonlinear refractive index is inside sample) to external selffocusing (focal point is outside sample) [1]. In this case the nonlinear refractive index of the sample causes a reduction of the overall beam divergence and the effects of small ripples across the beam profile may be neglected.

In our experiments we determine the reduction of beam divergence by comparing the beam diameters
(FWHM) of two pulses at a certain distance behind the sample position, where one pulse passes through the sample and the other pulse is bypassed. Our timeresolved measurements of the beam diameters with a streak camera and a two dimensional readout system allow the study of the instantaneous and transient contributions to the nonlinear refractive index $[12,18$, 20]. The effective time-averaged and the time-resolved nonlinear refractive index of benzene are measured with picosecond light pulses of a ruby laser. The reported time-averaged nonlinear refractive index coefficients $\bar{n}_{2}$ of benzene vary by a factor of ten in the region between $\bar{n}_{2}=1.4 \times 10^{-21} \mathrm{~m}^{2} \mathrm{~V}^{-2}$ and $1 \times 10^{-20} \mathrm{~m}^{2} \mathrm{~V}^{-2}$ [21-27] (see Table 1, $n_{2}[\mathrm{SI}]=\frac{1}{9}$ $\times 10^{-8} n_{2}[\mathrm{esu}]$ [6]).

\section{Theory}

The refractive index $n$ comprises a linear part $n_{\mathrm{L}}$ and a nonlinear part $\Delta n$, i.e. $n=n_{\mathrm{L}}+\Delta n$. The nonlinear part is composed of an electronic contribution, $\Delta n_{\mathrm{e}}$, which initantaneously follows the pulse intensity, and molecular contributions, $\Delta n_{\mathrm{m}}$, with transient response. The electronic part is given by

$\Delta n_{\mathrm{e}}=\gamma_{\mathrm{e}} I_{\mathrm{L}}=\frac{1}{2} n_{2 \mathrm{e}}\left|E_{\mathrm{OL}}\right|^{2}=\frac{n_{2 \mathrm{e}}}{n_{\mathrm{L}} \varepsilon_{0} c_{0}} I_{\mathrm{L}}$

where $I_{\mathrm{L}}=n_{\mathrm{L}} \varepsilon_{0} c_{0}\left|E_{\mathrm{oL}}\right|^{2} / 2$ is the light intensity, $E_{0}$ is the amplitude of the electrical field strength, $\varepsilon_{0}$ is the permittivity of vacuum, and $c_{0}$ is the speed of light in empty space. $\gamma_{e}$ is the electronic intensity coefficient 
Table 1. Reported nonlinear refractive index and orientational relaxation values of benzene at room temperature

\begin{tabular}{llll}
\hline Parameter & Technique & Wavelength & Ref. \\
\hline $\bar{n}_{2}=5.9 \times 10^{-21} \mathrm{~m}^{2} \mathrm{~V}^{-2}$ & Optical Kerr effect & $500 \mathrm{~nm}$ and $694.3 \mathrm{~nm}$ & 21,34 \\
$\bar{n}_{2}=1.0 \times 10^{-20} \mathrm{~m}^{2} \mathrm{~V}^{-2}$ & Time-integrated interference & $694.3 \mathrm{~nm}$ & 22 \\
$\bar{n}_{2}=1.4 \times 10^{-21} \mathrm{~m}^{2} \mathrm{~V}^{-2}$ & Optical Kerr effect & $694.3 \mathrm{~nm}$ & 23 \\
$\bar{n}_{2}=3.2 \times 10^{-21} \mathrm{~m}^{2} \mathrm{~V}^{-2}$ & Raman induced Kerr effect & $580 \mathrm{~nm}$ and $550 \mathrm{~nm}$ & 25 \\
$n_{2, \mathrm{st}}=6.7 \times 10^{-21} \mathrm{~m}^{2} \mathrm{~V}^{-2}$ & Optical Kerr effect & $1.06 \mu \mathrm{m}$ and $530 \mathrm{~nm}$ & 26 \\
$\bar{n}_{2}=3.0 \times 10^{-21} \mathrm{~m}^{2} \mathrm{~V}^{-2}$ & External self-focusing & $694.3 \mathrm{~nm}$ & This work \\
$n_{2 \mathrm{e}}=2.6 \times 10^{-21} \mathrm{~m}^{2} \mathrm{~V}^{-2}$ & Third harmonic generation & $1.89 \mu \mathrm{m}$ & 32 \\
$n_{2 \mathrm{e}}=4.9 \times 10^{-22} \mathrm{~m}^{2} \mathrm{~V}^{-2}$ & Three wave mixing & $555 \mathrm{~nm}$ & 33 \\
$n_{2 \mathrm{e}}=5.1 \times 10^{-22} \mathrm{~m}^{2} \mathrm{~V}^{-2}$ & Three wave mixing & $545 \mathrm{~nm}$ & 34 \\
$n_{2 \mathrm{e}}=3 \times 10^{-22} \mathrm{~m}^{2} \mathrm{~V}^{-2}$ & Field-induced SHG & $1.06 \mu \mathrm{m}$ & 35 \\
$n_{2 \mathrm{e}}=5.7 \times 10^{-22} \mathrm{~m}^{2} \mathrm{~V}^{-2}$ & Two-beam interference & $532 \mathrm{~nm}$ and $570 \mathrm{~nm}$ & 36 \\
$n_{2 \mathrm{e}}=2.6 \times 10^{-22} \mathrm{~m}^{2} \mathrm{~V}^{-2}$ & Third harmonic generation & $1.054 \mu \mathrm{m}$ & 37 \\
$n_{2 \mathrm{e}}=8.4 \times 10^{-23} \mathrm{~m}^{2} \mathrm{~V}^{-2}$ & fs optical Kerr effect & $620 \mathrm{~nm}$ & 38 \\
$\tau_{\mathrm{o}}=2 \mathrm{ps}$ & Raman linewidth & & 40 \\
$\tau_{\mathrm{o}}=4 \mathrm{ps}$ & Viscosity & & 41 \\
$\tau_{\mathrm{o}}=2.6 \mathrm{ps}$ & Raman linewidth & & 42 \\
$\tau_{\mathrm{o}}=2.8 \mathrm{ps}$ & Raman linewidth & 43 \\
$\tau_{\mathrm{o}}=2.9 \mathrm{ps}$ & Rayleigh linewidth & & 44,45 \\
$\tau_{\mathrm{o}}=3.05 \mathrm{ps}$ & Rayleigh linewidth & & 46 \\
$\tau_{\mathrm{o}}=3.14 \mathrm{ps}$ & Raman linewidth & & 47 \\
$\tau_{\mathrm{o}}=2.5 \mathrm{ps}$ & Raman linewidth & & \\
\hline
\end{tabular}

and $n_{2 \mathrm{e}}$ is the electronic field coefficient of the nonlinear refractive index. The molecular part consists of molecular orientation (optical Kerr effect), of molecular redistribution (librations), pressure (electrostriction), and thermal contributions $[12,28]$. It is given by

$$
\begin{aligned}
\Delta n_{\mathrm{m}}= & \sum_{i} \Delta n_{\mathrm{m}, i}=\sum_{i} \frac{n_{2, i}}{n_{\mathrm{L}} \varepsilon_{0} c_{0}} \frac{1}{\tau_{i}} \int_{-\infty}^{t} I_{\mathrm{L}}\left(t^{\prime}\right) \\
& \times \exp \left[-\left(t-t^{\prime}\right) / \tau_{i}\right] d t^{\prime}
\end{aligned}
$$

where $\tau_{i}$ is the response time of component $i$. The most important molecular contribution in liquids excited with picosecond light pulses is due to the optical Kerr effect $[12,21,28]$. Taking only this term into account, (2) reduces to

$$
\Delta n_{\mathrm{o}}=\frac{n_{2 \mathrm{o}}}{n_{\mathrm{L}} \varepsilon_{0} c_{0}} \frac{1}{\tau_{\mathrm{o}}} \int_{-\infty}^{t} I_{\mathrm{L}}\left(t^{\prime}\right) \exp \left[-\left(t-t^{\prime}\right) / \tau_{\mathrm{o}}\right] d t^{\prime},
$$

$\tau_{\mathrm{o}}$ is the reorientational relaxation time. In our discussions

$$
\begin{aligned}
\Delta n= & \Delta n_{\mathrm{e}}+\Delta n_{\mathrm{o}}=\frac{1}{n_{\mathrm{L}} \varepsilon_{0} c_{0}}\left\{n_{2 \mathrm{e}} I_{\mathrm{L}}(t)+\frac{n_{2 \mathrm{o}}}{\tau_{\mathrm{o}}}\right. \\
& \left.\times \int_{-\infty}^{t} I_{\mathrm{L}}\left(t^{\prime}\right) \exp \left[-\left(t-t^{\prime}\right) / \tau_{\mathrm{o}}\right] d t^{\prime}\right\} \\
= & \frac{n_{2, \mathrm{st}} I_{\mathrm{L}}(t)}{n_{\mathrm{L}} \varepsilon_{0} c_{0}}\left\{\frac{n_{2 \mathrm{e}}}{n_{2, \mathrm{st}}}+\frac{n_{2 \mathrm{o}}}{n_{2, \mathrm{st}} \tau_{\mathrm{o}}}\right. \\
& \left.\times \int_{-\infty}^{t} \frac{I_{\mathrm{L}}\left(t^{\prime}\right)}{I_{\mathrm{L}}(t)} \exp \left[-\left(t-t^{\prime}\right) / \tau_{\mathrm{o}}\right] d t^{\prime}\right\}
\end{aligned}
$$

is used. $n_{2, \mathrm{st}}=n_{2 \mathrm{e}}+n_{2 \mathrm{o}}$ is the total steady state intensity coefficient of the nonlinear refractive index.

In time-integrated measurements an effective field coefficient $\bar{n}_{2}$ will be determined by fitting the function

$\Delta n(t)=\frac{\bar{n}_{2}}{n_{\mathrm{L}} \varepsilon_{0} c_{0}} I_{\mathrm{L}}(t)$

to the experimental data.

For Gaussian pulses,

$I_{\mathrm{L}}(r, t)=I_{0 \mathrm{~L}} \exp \left[-\left(t / t_{0}\right)^{2}-(r / a)^{2}\right]$,

(4) reduces to

$\Delta n(r, t)=\frac{n_{2, \mathrm{st}} I_{\mathrm{OL}}}{n_{\mathrm{L}} \varepsilon_{0} c_{0}} \exp \left(-\frac{r^{2}}{a^{2}}\right) f(t)$

with

$$
\begin{aligned}
f(t)= & \exp \left(-\frac{t^{2}}{t_{0}^{2}}\right)\left\{\frac{n_{2 \mathrm{e}}}{n_{2, \mathrm{st}}}+\frac{n_{2 \mathrm{o}}}{n_{2, \mathrm{st}} \tau_{\mathrm{o}}}\right. \\
& \left.\times \int_{-\infty}^{t} \exp \left[-\frac{t^{\prime 2}-t^{2}}{t_{0}^{2}}-\frac{t-t^{\prime}}{\tau_{\mathrm{o}}}\right] d t^{\prime}\right\} .
\end{aligned}
$$

Under steady state conditions $f(t)$ becomes equal to $\exp \left(-t^{2} / t_{0}^{2}\right)$.

The light refraction in a nonlinear optical sample is illustrated in Fig. 1a and b. Only a principle plane is considered. The nonlinear refractive index, $\Delta n$, of the sample reduces the input beam divergence $\theta_{\mathrm{i}}$ by $\theta_{\mathrm{sf}}$ to 

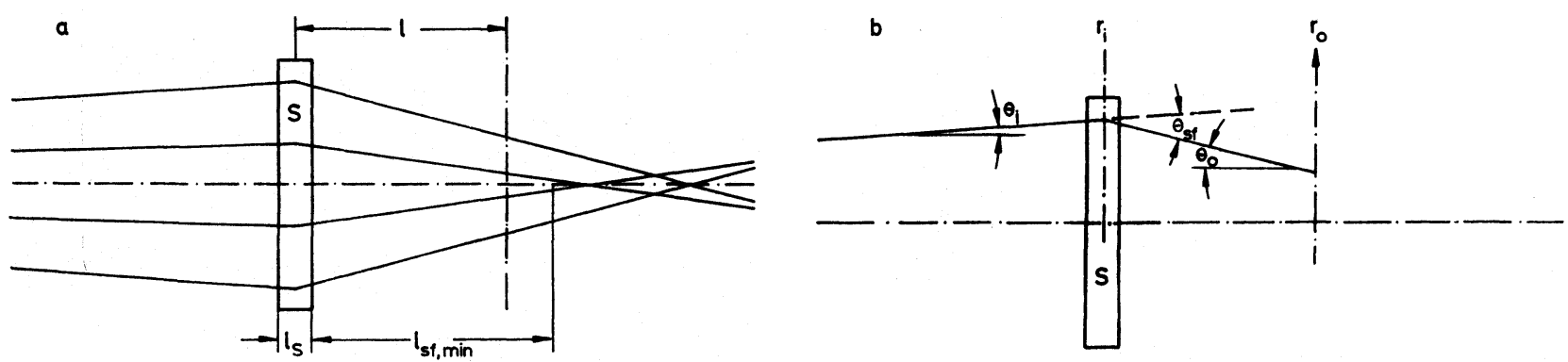

Fig. 1a, b. Schematic illustration of external self-focusing by light passage through sample $S$. a Ray bending leading to spherical aberration. b Indication of input divergence $\theta_{\mathrm{i}}$, output divergence $\theta_{\mathrm{o}}$, and ray deflection $\theta_{\mathrm{sf}}$ due to self-focusing action

an output beam divergence $\theta_{0}$. It is

$\theta_{\mathrm{o}}=\theta_{\mathrm{i}}+\theta_{\mathrm{sf}}$.

The input divergence is

$\theta_{\mathrm{i}}\left(r_{\mathrm{i}}\right)=\frac{r_{\mathrm{i}}}{R_{\mathrm{i}}}$

where $r_{\mathrm{i}}$ is the ray coordinate at the sample and $R_{\mathrm{i}}$ is the wavefront curvature radius of the input pulse at the sample. The change of beam divergence, $\theta_{\mathrm{sf}}$, due to the self-focusing action is given by [Ref. 29, Fig. 27 and Eq. (138a)]:

$$
\begin{aligned}
\theta_{\mathrm{sf}}=l_{\mathrm{s}} \frac{\partial \Delta n}{\partial r_{\mathrm{i}}} & =-\frac{2 l_{\mathrm{s}} r_{\mathrm{i}} n_{2, \mathrm{st}}}{n_{\mathrm{L}} \varepsilon_{0} c_{0} a_{\mathrm{i}}^{2}} I_{0 \mathrm{~L}} \exp \left(-\frac{r_{\mathrm{i}}^{2}}{a_{\mathrm{i}}^{2}}\right) f(t) \\
& =\frac{r_{\mathrm{i}}}{R_{\mathrm{sf}}\left(r_{\mathrm{i}}, t\right)}=\frac{r_{\mathrm{i}}}{R_{\mathrm{sf}, 0}} \exp \left(-\frac{r_{\mathrm{i}}^{2}}{a_{\mathrm{i}}^{2}}\right) f(t)
\end{aligned}
$$

where $l_{\mathrm{s}}$ is the sample length. $R_{\mathrm{sf}}\left(r_{\mathrm{i}}, t\right)$ is the change of the wavefront curvature radius due to self-focusing. The shortest wavefront radius occurs at $r=0 . R_{\mathrm{sf}, 0}$ is $r_{\mathrm{i}}$ is given by

$l_{\mathrm{sf}}^{-1}=\left|\frac{\theta_{\mathrm{o}}}{r_{\mathrm{i}}}\right|=\left|R_{\mathrm{i}}^{-1}+R_{\mathrm{sf}}^{-1}\left(r_{\mathrm{i}}, t\right)\right|$.

$l_{\mathrm{sf}}$ has its minimum, $l_{\mathrm{sf}, \min }$, at the beam center $\left(r_{\mathrm{i}}=0\right)$. At distances $l>l_{\text {sf, min }}$ some rays cross (Fig. 1a) and it becomes difficult to calculate the spatial intensity distribution at $l$. Our calculations restrict to $l<l_{\mathrm{sf} \text {, min }}$ and the intensity distribution at $l$ is given by

$I_{\mathrm{L}, \mathrm{sf}}\left(r_{0}, t\right)=\frac{r_{\mathrm{i}}}{r_{\mathrm{o}}} \frac{\partial r_{\mathrm{i}}}{\partial r_{\mathrm{o}}} I_{0 \mathrm{~L}} \exp \left(-\frac{r_{\mathrm{i}}^{2}}{a_{\mathrm{i}}^{2}}-\frac{t^{2}}{t_{0}^{2}}\right)$

with

$r_{\mathrm{o}}=r_{\mathrm{i}}+\theta_{\mathrm{o}} l=r_{\mathrm{i}}\left\{1+\left[\frac{1}{R_{\mathrm{i}}}+\frac{1}{R_{\mathrm{sf}}\left(r_{\mathrm{i}}, t\right)}\right] l\right\}$

and

$\frac{\partial r_{\mathrm{i}}}{\partial r_{\mathrm{o}}}=\left[1+\frac{l}{R_{\mathrm{i}}}+\frac{l}{R_{\mathrm{sf}}\left(r_{\mathrm{i}}, t\right)}\left(1-\frac{2 r_{\mathrm{i}}^{2}}{a_{\mathrm{i}}^{2}}\right)\right]^{-1}$.

Insertion of (14) and (15) into (13) results in

$I_{\mathrm{L}, \mathrm{sf}}\left(r_{\mathrm{o}}, t\right)=\frac{I_{\mathrm{oL}} \exp \left(-\frac{r_{\mathrm{i}}^{2}}{a_{\mathrm{i}}^{2}}-\frac{t^{2}}{t_{0}^{2}}\right)}{\left[1+\frac{l}{R_{\mathrm{i}}}+\frac{l}{R_{\mathrm{sf}}\left(r_{\mathrm{i}}, t\right)}\right]\left[1+\frac{l}{R_{\mathrm{i}}}+\frac{l}{R_{\mathrm{sf}}\left(r_{\mathrm{i}}, t\right)}\left(1-\frac{2 r_{\mathrm{i}}^{2}}{a_{\mathrm{i}}^{2}}\right)\right]}$

and the spatial intensity distribution function $s_{\mathrm{sf}}\left(r_{0}, t\right)$ at time $t$ is

$s_{\mathrm{sf}}\left(r_{\mathrm{o}}, t\right)=\frac{I_{\mathrm{L}, \mathrm{sr}}\left(r_{\mathrm{o}}, t\right)}{I_{\mathrm{L}, \mathrm{sr}}(0, t)}=\frac{\exp \left(-\frac{r_{\mathrm{i}}^{2}}{a_{\mathrm{i}}^{2}}\right)\left[1+\frac{l}{R_{\mathrm{i}}}+\frac{l}{R_{\mathrm{sf}}(0, t)}\right]^{2}}{\left[1+\frac{l}{R_{\mathrm{i}}}+\frac{l}{R_{\mathrm{sr}}\left(r_{\mathrm{i}}, t\right)}\right]\left[1+\frac{l}{R_{\mathrm{i}}}+\frac{l}{R_{\mathrm{sr}}\left(r_{\mathrm{i}}, t\right)}\left(1-\frac{2 r_{\mathrm{i}}^{2}}{a_{\mathrm{i}}^{2}}\right)\right]}$.

the steady-state wavefront radius at $r=0$ and $t=0$. It is

$R_{\mathrm{sf}, 0}=-\frac{n_{\mathrm{L}} \varepsilon_{0} c_{0} a_{\mathrm{i}}^{2}}{2 l_{\mathrm{s}} n_{2, \mathrm{st}} I_{\mathrm{OL}}}$.

As is shown in Fig. 1a, there exists no common external self-focusing point because of the spatial Gaussian dependence of $\theta_{\mathrm{sf}}$ (spherical aberration [1]). The external self-focusing length, $l_{\mathrm{sf}}$, of a ray at position
The spatial energy density $w_{\mathrm{sf}}\left(r_{\mathrm{o}}\right)$ is

$w_{\mathrm{sf}}\left(r_{\mathrm{o}}\right)=\int_{-\infty}^{\infty} I_{\mathrm{L}, \mathrm{sf}}\left(r_{\mathrm{o}}, t\right) d t$

and the spatial energy distribution function is

$s_{\mathrm{w}, \mathrm{sf}}\left(r_{\mathrm{o}}\right)=\frac{w_{\mathrm{sf}}\left(r_{\mathrm{o}}\right)}{w_{\mathrm{sf}}(0)}$ 
The intensity distribution of the pulse bypassing the sample is

$I_{\mathrm{L}, \mathrm{nsf}}\left(r_{\mathrm{o}}, t\right)=\frac{I_{0 \mathrm{~L}} \exp \left(-\frac{r_{\mathrm{i}}^{2}}{a_{\mathrm{i}}^{2}}-\frac{t^{2}}{t_{0}^{2}}\right)}{\left(1+\frac{l}{R_{\mathrm{i}}}\right)^{2}}$

with

$r_{\mathrm{o}}=r_{\mathrm{i}}\left(1+\frac{l}{R_{\mathrm{i}}}\right)$

and the intensity distribution function, $s_{\mathrm{nsf}}\left(r_{\mathrm{o}}, t\right)$, is

$s_{\mathrm{nsf}}\left(r_{\mathrm{o}}, t\right)=\frac{I_{\mathrm{L}, \mathrm{nsf}}\left(r_{\mathrm{o}}, t\right)}{I_{\mathrm{L}, \mathrm{nsf}}(0, t)}=\exp \left(-\frac{r_{\mathrm{i}}^{2}}{a_{\mathrm{i}}^{2}}\right)=\exp \left(-\frac{r_{\mathrm{o}}^{2}}{a_{\mathrm{o}}^{2}}\right)$

with

$a_{\mathrm{o}}=a_{\mathrm{i}}\left(1+\frac{l}{R_{\mathrm{i}}}\right)$.

The intensity distribution function, $s_{\mathrm{nsf}}\left(r_{\mathrm{o}}, t\right)$, is equal to the energy distribution function, $s_{\mathrm{w}, \mathrm{nsf}}\left(r_{\mathrm{o}}\right)$. In the analysis $s_{\mathrm{sf}}\left(r_{\mathrm{o}}, t\right)$ and $s_{\mathrm{w}, \mathrm{sf}}\left(r_{\mathrm{o}}\right)$ as well as the spatial half-widths

$\Delta r_{\mathrm{sf}}(t)\left[s_{\mathrm{sf}}\left(\Delta r_{\mathrm{sf}}, t\right)=0.5\right]$

and

$\Delta r_{\mathrm{w}, \mathrm{sf}}\left[s_{\mathrm{w}, \mathrm{sf}}\left(\Delta r_{\mathrm{w}, \mathrm{sf}}\right)=0.5\right]$

are determined numerically and related to the non-selffocused situation. The calculated ratios of $\Delta r_{\text {sf }}(t) / \Delta r_{\text {nsf }}$ and $\Delta r_{\mathrm{w}, \mathrm{sf}} / \Delta r_{\mathrm{nsf}}$ are compared with experimental results $\left[\Delta r_{\text {nsf }}\right.$ is the spatial half-width of the non-selffocused beam (HWHM)].

\section{Experimental Arrangement}

The experimental setup is shown in Fig. 2. Trains of pico-second light pulses are generated in a passively mode-locked ruby laser (pulse duration $\Delta t_{\mathrm{L}} \simeq 35 \mathrm{ps}$, laser wavelength $\lambda_{\mathrm{L}}=694.3 \mathrm{~nm}$ ) [30]. Single pulses are selected with a Pockels cell shutter and the separated pulses are increased in energy in a ruby amplifier. The laser pulses are split into two parts. One part serves as reference and is passed directly to the streak camera SC. The second part passes through the sample cell $\mathrm{S}$ before it is directed to the streak camera. The peak intensity of this pulse is determined by energy transmission measurement (photodetectors PD 1 and PD 2) through the saturable absorber DDI [31] (small signal transmission $T_{0}=0.03$ ). The optical paths of the two beams are adjusted so that they simultaneously reach the input slit of the streak camera (Hamamatsu type C1587 with fast streak plug-in type M 1952, highest

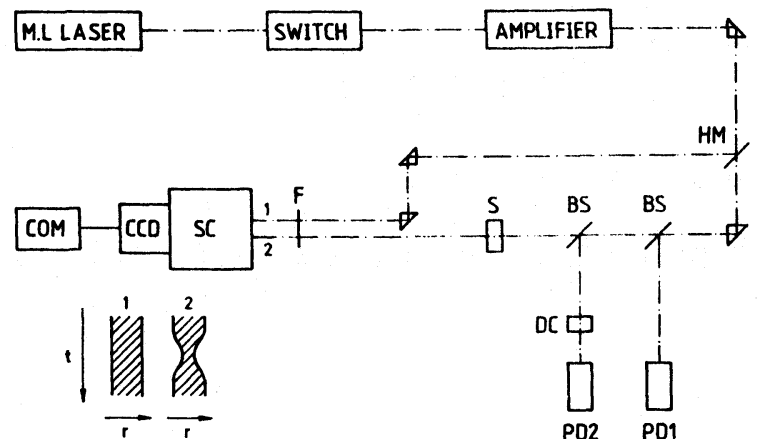

Fig. 2. Experimental set-up. HM, 50\% mirror. BS, beam splitters. DC, saturable absorber cell for peak intensity detection. PD 1, PD 2, photodetectors. S, sample cell. F, filters. SC, streak camera. CCD, two-dimensional image-intensified CCD camera. COM, personal computer. Inset below streak camera illustrates spatial shapes of the non-self-focused beam 1 and of the self-focused beam 2

time resolution is approximately $2 \mathrm{ps}$ ). The narrow central stripes of the pulses passing through the input slit are temporally resolved by the streak camera. The time-resolved spatial intensity distributions of both pulses at the streak camera output screen are recorded with an intensified two-dimensional CCD camera (Proxitronic type PC 1450LL). The CCD images are analysed with an image processing system (Imaging Technology type PC VISION plus Frame Grabber) and a personal computer (Olivetti M 24). The picture analysis allows the determination of the time-resolved spatial intensity distributions $I_{\mathrm{L}}\left(r_{\mathrm{o}}, t\right)$ and of the timeresolved beam radii $\Delta r(t)$. The temporal integration of the pictures gives the spatial energy density distributions $w\left(r_{0}\right)$ and the time-integrated beam radii $\Delta r_{w}$.

The experimental parameters of the external selffocusing measurements are collected in Table 2.

\section{Experimental Results}

The experimental time-integrated beam narrowing is displayed in Fig. 3. The ratios of the beam radii $\Delta r_{\text {w, sf }} / \Delta r_{\text {nsf }}$ are plotted versus the normalized input pump pulse peak intensity $I_{0 L} / a_{i}^{2}$. The curves in Fig. 3 are calculated for various effective nonlinear field coefficients $\bar{n}_{2}$. The best-fitting value is $\bar{n}_{2}=3.0$ $\times 10^{-21} \mathrm{~m}^{2} \mathrm{~V}^{-2}$ (Table 2). This value is in the range of previously reported $\bar{n}_{2}$-values [21-27] and it agrees very well with $\bar{n}_{2}=3.2 \times 10^{-21} \mathrm{~m}^{2} \mathrm{~V}^{-2}$ of [25], which was determined by Raman-induced Kerr-effect measurements.

The electronic field coefficient $n_{2 \mathrm{e}}$ has been reported in the range between $8.4 \times 10^{-23} \mathrm{~m}^{2} \mathrm{~V}^{-2}$ and $2.6 \times 10^{-21} \mathrm{~m}^{2} \mathrm{~V}^{-2}$ [32-38] (Table 1). The relation between $n_{2 \mathrm{e}}$ and the third-order nonlinear electronic 
Table 2. Experimental parameters and results

\begin{tabular}{|c|c|c|}
\hline Parameter & Value & Ref. \\
\hline \multicolumn{3}{|l|}{ Experimental parameter } \\
\hline Laser wavelength & $\lambda_{\mathrm{L}}=694.3 \mathrm{~nm}$ & \\
\hline Average pulse duration & $\Delta t_{\mathrm{L}}=35 \mathrm{ps}(\mathrm{FWHM})$ & \\
\hline Wavefront curvature & $R_{\mathrm{i}}=8.7 \mathrm{~m}$ & \\
\hline $1 / e$-beam radius & $a_{\mathrm{i}}=1.25 \mathrm{~mm}$ & \\
\hline Sample length & $l_{\mathrm{s}}=5 \mathrm{~cm}$ & \\
\hline Distance & $l=1.26 \mathrm{~m}$ & \\
\hline \multicolumn{3}{|l|}{ Benzene parameters } \\
\hline Linear refractive index & $n_{\mathrm{L}}=1.4982$ & a \\
\hline Average field coefficient & $\bar{n}_{2}=(3 \pm 0.3) \times 10^{-21} \mathrm{~m}^{2} \mathrm{~V}^{-2}$ & This work \\
\hline Electronic field coefficient & $n_{2 \mathrm{e}}=2.6 \times 10^{-22} \mathrm{~m}^{2} \mathrm{~V}^{-2}$ & 37 \\
\hline Orientational field coefficient & $n_{2 \mathrm{o}}=(2.8 \pm 0.3) \times 10^{-21} \mathrm{~m}^{2} \mathrm{~V}^{-2}$ & This work \\
\hline Steady-state field coefficient & $n_{2, \mathrm{st}}=(3.1 \pm 0.3) \times 10^{-21} \mathrm{~m}^{2} \mathrm{~V}^{-2}$ & This work \\
\hline Rotational relaxation time & $\tau_{\mathrm{o}}=3.1 \mathrm{ps}$ & 46 \\
\hline
\end{tabular}

${ }^{a}$ Extrapolated from [48]

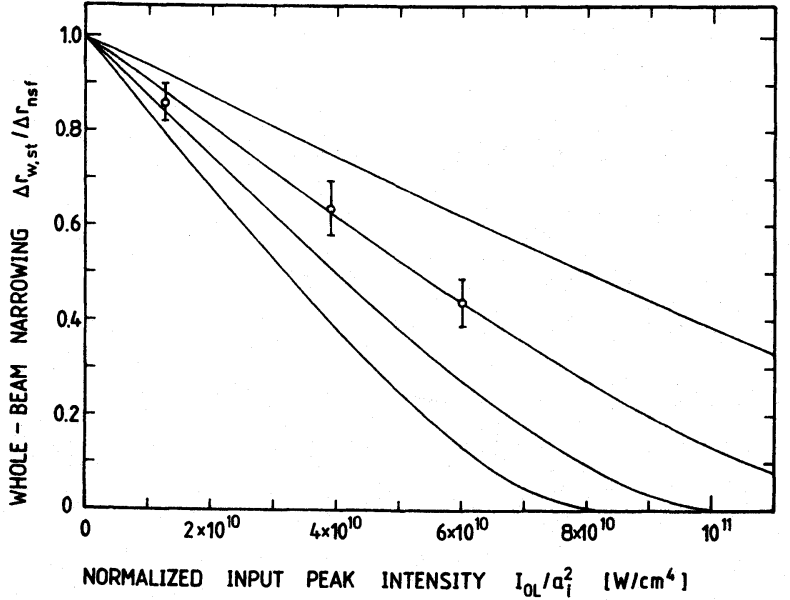

Fig. 3. Time-integrated beam narrowing versus input pump pulse peak intensity. The experimental parameters are listed in Table 2. The circles are measured. The curves are calculated for (1) $\bar{n}_{2}=2 \times 10^{-21} \mathrm{~m}^{2} \mathrm{~V}^{-2}$, (2) $\bar{n}_{2}=3 \times 10^{-21} \mathrm{~m}^{2} \mathrm{~V}^{-2}$, (3) $\bar{n}_{2}=4$ $\times 10^{-21} \mathrm{~m}^{2} \mathrm{~V}^{-2}$, and (4) $n_{2}=5 \times 10^{-21} \mathrm{~m}^{2} \mathrm{~V}^{-2}$

susceptibility $\chi_{x x x x, e}^{(3)}$ is given by [39]:

$$
\begin{aligned}
n_{2 \mathrm{e}} & =\frac{3}{n_{\mathrm{L}}} \chi_{x x x x, \mathrm{e}}^{(3)}\left(-\omega_{\mathrm{L}} ; \omega_{\mathrm{L}},-\omega_{\mathrm{L}}, \omega_{\mathrm{L}}\right) \\
& =\frac{3}{n_{\mathrm{L}}} \chi_{x x x x, \mathrm{e}}^{(3)}\left(-3 \omega_{\mathrm{L}} ; \omega_{\mathrm{L}}, \omega_{\mathrm{L}}, \omega_{\mathrm{L}}\right) .
\end{aligned}
$$

The last equality is valid if the third harmonic nonlinear susceptibility

$\chi_{\mathrm{THG}}^{(3)}=\chi_{x x x x, \mathrm{e}}^{(3)}\left(-3 \omega_{\mathrm{L}}, \omega_{\mathrm{L}}, \omega_{\mathrm{L}}, \omega_{\mathrm{L}}\right)$

is not enhanced resonantly. [For esu units the relation is $\left.n_{2 \mathrm{e}}=\left(12 \pi / n_{\mathrm{L}}\right) \chi_{x x x x, \mathrm{e}}^{(3)}.\right]$ The effective field coefficient $\bar{n}_{2}$ is approximately a factor of ten larger than the electronic field coefficient $n_{2 \mathrm{e}}$ of $[35,37]$ indicating

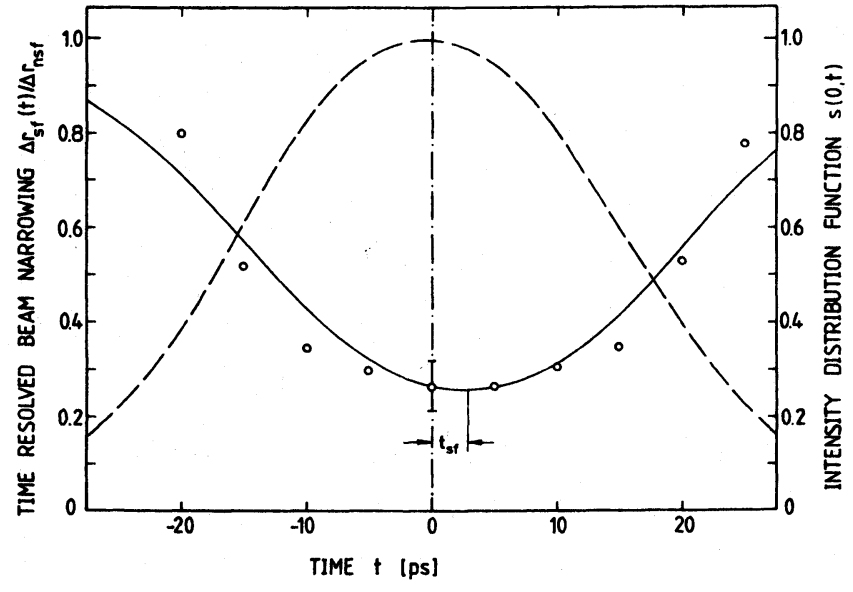

Fig. 4. Time-resolved beam narrowing $\Delta r_{\mathrm{sf}}(t) / \Delta r_{\text {nsf }}$ of a laser pulse. The dashed curve shows the input temporal pulse shape (streak camera picture of non-self-focused beam). The input pulse peak intensity is $I_{0 \mathrm{~L}}=1.1 \times 10^{9} \mathrm{~W} / \mathrm{cm}^{2}$ and the pulse duration is $\Delta t_{\mathrm{L}}=35 \mathrm{ps}$. The circles show digitized beam narrowing ratios. The solid curve is calculated with the parameters of Table 2

that $\bar{n}_{2}$ is mainly determined by the orientational field coefficient $n_{20}$.

In Fig. 4 the time-resolved beam narrowing is shown for a single shot with a pump pulse peak intensity of $I_{0 \mathrm{~L}} \simeq 1.1 \times 10^{9} \mathrm{~W} / \mathrm{cm}^{2}$ and a pulse duration of $\Delta t_{\mathrm{L}} \simeq 35 \mathrm{ps}$. $\Delta r_{\mathrm{sf}}(t) / \Delta r_{\mathrm{nsf}}$ is plotted versus time. The dashed curve shows the temporal pulse shape of the non-self-focused pulse. The minimum width of the selffocused beam occurs slightly behind the pulse maximum. The temporal shift $t_{\mathrm{sf}}$ of the minimum width behind the pulse maximum is determined by the response time $\tau_{\mathrm{o}}$ of the molecular nonlinear refractive index contribution. For small shifts $t_{\mathrm{sf}}$ is slightly less than $\tau_{\mathrm{o}}$ (for large shifts $t_{\mathrm{sf}}$ becomes considerably less than $\tau_{0}$, see Fig. 6). The solid curve in Fig. 4 is 
calculated with the $n_{2 \mathrm{e}}, n_{2 \mathrm{o}}$, and $\tau_{\mathrm{o}}$ values listed in Table 2. $n_{20}$ is a factor of ten larger than $n_{2 \mathrm{e}} \cdot n_{2, \mathrm{st}}=n_{2 \mathrm{e}}$ $+n_{20}$ is approximately equal to $\bar{n}_{2}$. This fact indicates that external self-focusing in benzene occurs under nearly steady-state conditions for ruby laser pulses of approximately $30 \mathrm{ps}$ to $40 \mathrm{ps}$ duration. The orientational relaxation time of benzene of $\tau_{0} \simeq 3.1 \mathrm{ps}$ [40-47] (Table 1) is approximately a factor of ten shorter than the pulse duration.

\section{Computer Simulations}

The dependence of the external self-focusing on various pump pulse parameters and material parameters is analysed numerically in the following.

In Fig. 5 the time-integrated beam narrowing, $\Delta r_{\mathrm{w}, \mathrm{sf}} / \Delta r_{\mathrm{nsf}}$, is plotted versus the steady-state focusing strength

$\kappa_{\mathrm{sf}}=\left|\frac{l}{R_{\mathrm{sf}, \mathrm{o}}}\right|=\frac{2 l l_{\mathrm{s}} n_{2, \mathrm{st}} I_{\mathrm{OL}}}{n_{\mathrm{L}} \varepsilon_{0} c_{0} a_{\mathrm{i}}^{2}}$

for various transient situations. The time ratio $\tau_{0} / t_{0}$ is varied. The curves belong to $R_{\mathrm{i}}=\infty$ and $n_{20} / n_{2, \mathrm{st}}=1$. The beam radius $a_{\mathrm{i}}$ has no influence. The self-focusing action is strongest for the steady-state situation $\left(\tau_{0} / t_{0} \rightarrow 0\right)$.

The time-resolved beam narrowing, $\Delta r_{\mathrm{sf}}(t) / \Delta r_{\mathrm{nsf}}$, is illustrated in Fig. 6. The input pulse has a Gaussian shape. It is indicated by the dotted curve. The time ratio $\tau_{\mathrm{o}} / t_{0}$ is varied. The solid curves belong to $\kappa_{\mathrm{st}}=0.5$, $R_{\mathrm{i}}=\infty$, and $n_{2 \mathrm{o}} / n_{2, \mathrm{st}}=1$ with different $\tau_{\mathrm{o}} / t_{0}$ values. The dashed curve belongs to $\tau_{\mathrm{o}} / t_{0}=0$. The dash-dotted curve is calculated for $\kappa_{\mathrm{st}}=0.5, R_{\mathrm{i}}=\infty, n_{20} / n_{2, \mathrm{st}}=0.8$,

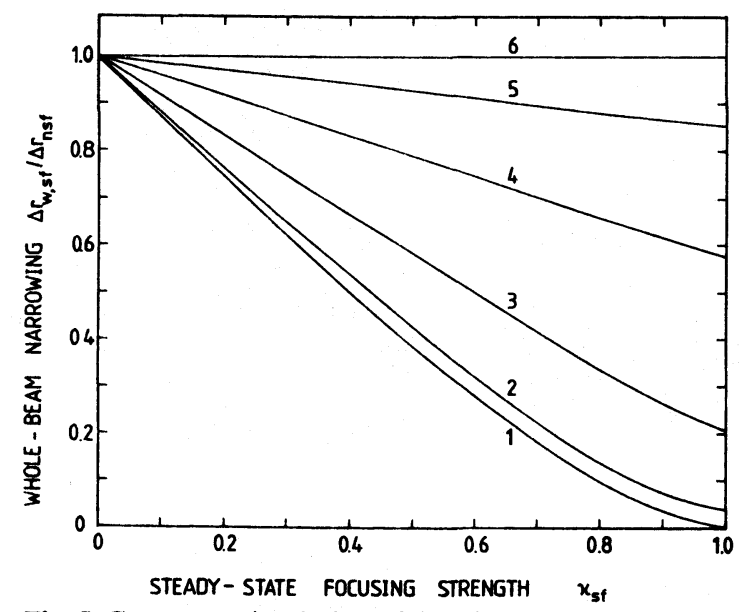

Fig. 5. Computer simulation of time-integrated beam narrowing, $\Delta r_{\mathrm{w}, \mathrm{sf}} / \Delta r_{\mathrm{nsf}}$, versus steady-state focusing strength $\kappa_{\mathrm{sf}}=l / R_{\mathrm{sf}, 0}$. Fixed parameters are: $R_{\mathrm{i}}=\infty$ and $n_{2 \mathrm{e}}=0$. The curves belong to $\tau_{\mathrm{o}} / t_{0}=0(1), 0.3(2), 1(3), 3(4), 10(5)$, and 100(6). Curves are independent of input beam radius $a_{\mathrm{i}}$

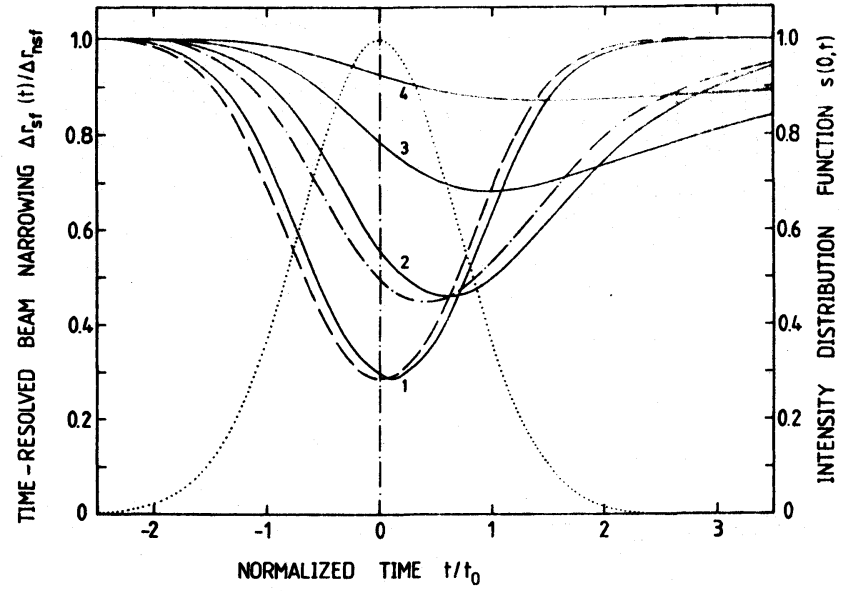

Fig. 6. Computer simulation of time-resolved beam narrowing, $\Delta r_{\text {sf }}(t) / \Delta r_{\text {nsf }}$, versus time. The dotted curve shows the temporal input pulse shape. Solid curves: $R_{\mathrm{i}}=\infty, n_{2 \mathrm{e}}=0, \kappa_{\mathrm{st}}=l / R_{\mathrm{sf}, 0}=0.5$ with $\tau_{\mathrm{o}} / t_{0}=0.1(1), 1(2), 3(3)$, and 10(4). Dashed curve: $R_{\mathrm{i}}=\infty$, $n_{2 \mathrm{o}}=0$, and $\kappa_{\mathrm{st}}=0.5$. Dash-dotted curve: $R_{\mathrm{i}}=\infty, n_{2 \mathrm{e}}=0.2 n_{2, \mathrm{st}}$, $\kappa_{\mathrm{st}}=0.5$, and $\tau_{\mathrm{o}} / t_{0}=1$

and $\tau_{0} / t_{0}=1$. The beam-width minimum shifts to longer delays with increasing response time and increasing $n_{20} / n_{2, \text { st }}$ values. The beam narrowing effect reduces with increasing response time.

\section{Conclusions}

The analysis of the time-integrated and time-resolved beam narrowing caused by external self-focusing offers a method to determine the nonlinear refractive indices of materials. The whole-beam narrowing provides an effective nonlinear refractive index coefficient $\bar{n}_{2}$, while time-resolved beam narrowing measurements allow the separation of fast electronic and slow molecular nonlinear refractive index contributions. Response times in the temporal region of $0 \leqq \tau_{0} \leqq 2 t_{0}$ may be determined by the time-resolved beam narrowing analysis (Fig. 6).

Acknowledgements. The authors thank the Deutsche Forschungsgemeinschaft for financial support and the Rechenzentrum of the University of Regensburg for the allocation of computer time.

\section{References}

1. S.A. Akhmanov, R.V. Khokhlov, A.P. Sukhorukov: Laser Handbook Vol. II, ed. by F.T. Arrechi, E.O. Schulz-Dubois (North-Holland, Amsterdam 1972) Ch. E3

2. Y.R. Shen: Prog. Quant. Electron. 4, 1 (1975)

3. J.H. Marburger: Prog. Quant. Electron. 4, 35 (1975)

4. Y.R. Shen: The Principles of Nonlinear Optics (Wiley, New York 1984)

5. J.F. Reintges: Nonlinear Optical Parametric Processes in Liquids and Gases (Academic, Orlando 1984)

6. W. Koechner: Solid State Laser Engineering 2nd ed. Springer Ser. Opt. Sci. Vol. 1 (Springer, Berlin, Heidelberg 1988) 
7. V.I. Bespalov, V.I. Talanov: JETP Lett. 3, 307 (1966)

8. A.J. Campillo, S.L. Shapiro, B.R. Suydam: Appl. Phys. Lett. 24, 178 (1974)

9. R.G. Brewer, J.R. Lifsitz, E. Garmire, R.Y. Chiao, C.H. Townes: Phys. Rev. 166, 326 (1968)

10. T.K. Gustafson, J.P. Taran, H.A. Haus, J.R. Lifsitz, P.L. Kelley: Phys. Rev. 177, 306 (1969)

11. M.T. Loy, Y.R. Shen: Phys. Rev. Lett. 22, 994 (1969)

12. O. Svelto: Prog. Opt. 12, 1 (1974)

13. J.B. Grun, A.K. McQuillan, B.P. Stoicheff: Phys. Rev. 180, 61 (1969)

14. N. Bloembergen, P. Lallemand: In Physics of Quantum Electronics, ed. by P.L. Kelley, B. Lax, N.P. Tannenwald (McGraw-Hill, New York 1966)

15. N. Bloembergen: IEEE J. Quant. Electron. QE-10, 375 (1974)

16. O. Rahn, M. Maier: Phys. Rev. A9, 1427 (1974)

17. A.H. Piekara, T.K. Gustafson: Opt. Commun. 7, 197 (1973)

18. F. Shimizu: IBM J. Res. Dev. 17, 286 (1973)

19. A.N. Arbatskaya: In Stimulated Raman Scattering ed. by N.G. Basov, Proc. P.N. Lebedev Physics Institute, Vol. 99 (Consultants Bureau, New York 1982) p. 1

20. M.M.T. Loy, Y.R. Shen: IEEE J. Quant. Electron. QE-9, 409 (1973)

21. G. Mayer, F. Gires: C.R. Acad. Sci. (Paris) 258, 2039 (1964)

22. A.P. Veduta, B.P. Kirsanov: Sov. Phys. JETP 27, 736 (1968)

23. M. Paillette: Ann. Phys. (Paris) 4, 671 (1969)

24. A. Owyoung: Appl. Phys. Lett. 26, 168 (1975)

25. J.J. Song, M.D. Levenson: J. Appl. Phys. 48, 3496 (1977)

26. P.P. Ho, R.R. Alfano: Phys. Rev. A20, 2170 (1979)

27. W.L. Smith: In CRC Handbook of Laser Science and Technology Vol. III, Part 1, ed. by M.J. Weber (CRC Press, Boca Raton, Florida 1986) p. 259
28. J. Reintjes, R.L. Carman: Phys. Rev. Lett. 28, 1697 (1972)

29. A. Penzkofer: Prog. Quant. Electron. 12, 291 (1988)

30. P. Sperber, A. Penzkofer: Opt. Commun. 54, 160 (1985)

31. W. Blau, R. Reber, A. Penzkofer: Opt. Commun. 43, 210 (1982)

32. J.P. Hermann: Opt. Commun. 9, 74 (1973)

33. M.D. Levenson, N. Bloembergen: J. Chem. Phys. 60, 1323 (1974)

34. M.D. Levenson, N. Bloembergen: Phys. Rev. B 10, 4447 (1974)

35. B.F. Levine, C.G. Bethea: J. Chem. Phys. 63, 2666 (1975)

36. A. Owyoung: Opt. Commun. 16, 266 (1976)

37. M. Thalhammer, A. Penzkofer: Appl. Phys. B 32, 137 (1983)

38. J. Etchepare, G. Grillon, R. Muller, A. Orszag: Opt. Commun. 34, 269 (1980)

39. A. Penzkofer, W. Leupacher: Opt. Quant. Electron. 20, 227 (1988)

40. A.V. Rakov: In Research in Molecular Spectroscopy, Proc. P.N. Lebedev Physics Institute, Vol. 77, ed. by D.V. Skobel'tsyn (Consultants Bureau, New York 1965) p. 109

41. M.J. Colles: Opt. Commun. 1, 169 (1969)

42. F.J. Bartoli, T.A. Litowitz: J. Chem. Phys. 56, 404 (1972)

43. K.T. Gillen, S.E. Griffiths: Chem. Phys. Lett. 17, 359 (1972)

44. G.R. Alms, D.R. Bauer, J.I. Brauman, R. Pecora: J. Chem. Phys. 58, 5570 (1973)

45. D.R. Bauer, G.R. Alms, J.I. Brauman, R. Pecora: J. Chem. Phys. 61, 2255 (1974)

46. K.L. Oehme, F. Seifert, G. Rudakoff, W. Carius, W. Hölzer, O. Schröter: Chem. Phys. 92, 169 (1985)

47. D. Samios, T. Dorfmüller: Chem. Phys. Lett. 117, 165 (1985)

48. Landolt-Börnstein, 6th ed., ed. by K.A. Hellwege, A.M. Hellwege (Springer, Berlin, Heidelberg 1962) Vol. 2, Part 8 